\title{
Sub- and superoptimality principles and construction of almost optimal strategies for differential games in Hilbert spaces
}

Andrzej Święch

Abstract We prove sub- and superoptimality principles of dynamic programming and show how to use the theory of viscosity solutions to construct almost optimal strategies for two-player, zero-sum differential games driven by abstract evolution equations in Hilbert spaces.

\section{Introduction}

The main purpose of this paper is to show how Isaacs equations and the theory of viscosity solutions can be used to construct nearly optimal strategies for two-player, zero-sum differential games driven by abstract evolution equations in a Hilbert space. Let us briefly describe the game we have in mind. Let $H$ be a real, separable Hilbert space and $T>0$ be a fixed time horizon. For an initial time $t \in[0, T]$ and $x \in H$, the dynamics of the game are given by an evolution equation

$$
\left\{\begin{array}{l}
\frac{d}{d s} X(s)=A X(s)+b(s, X(s), W(s), Z(s)) \\
X(t)=x
\end{array}\right.
$$

where $A$ is a linear, densely defined maximal dissipative operator in $H, b:[0, T] \times$ $H \times \mathscr{W} \times \mathscr{Z} \rightarrow \mathbb{R}$ for some metric spaces $\mathscr{W}, \mathscr{Z}$, and

$$
\begin{gathered}
W \in M(t):=\{W:[t, T] \rightarrow \mathscr{W}, \text { strongly measurable }\}, \\
Y \in N(t):=\{Z:[t, T] \rightarrow \mathscr{Z}, \text { strongly measurable }\} .
\end{gathered}
$$

We will call $M(t)$ the set of controls for player I, and $N(t)$ the set of controls for player II.

School of Mathematics, Georgia Institute of Technology Atlanta, GA 30332, U.S.A. e-mail: swiech@math.gatech.edu 
This setting is very general. The state equation can be for instance a single equation or a system of controlled semilinear PDE. We recall that an operator $A$ is maximal dissipative if and only if it generates a semigroup of contractions $e^{t A}$, i.e. $\left\|e^{t A}\right\| \leq 1$.

The pay-off functional associated with the trajectory of the system given by 1 is

$$
J(t, x ; W(\cdot), Z(\cdot))=\int_{t}^{T} L(s, X(s), W(s), Z(s)) d s+h(X(T)) .
$$

Player I controls $W$ and wants to maximize $J$ over all choices of $Z$. Player II controls $Z$ and wants to minimize $J$ over all choices of $W$. The game is played in continuous time. Following Elliot and Kalton [8] we define admissible strategies for both players and consider the upper and lower versions of the game. In the lower game player II chooses $Z(s)$ knowing $W(s)$, and in the upper game player I chooses $W(s)$ knowing $Z(s)$.

Admissible strategies of player I:

$$
\Gamma(t):=\{\alpha: N(t) \rightarrow M(t), \text { nonanticipating }\}
$$

Admissible strategies of player II:

$$
\Delta(t):=\{\beta: M(t) \rightarrow N(t), \text { nonanticipating }\} .
$$

Strategy $\alpha$ (resp., $\beta$ ) is nonanticipating if whenever $Z_{1}(r)=Z_{2}(r)$ (resp., $W_{1}(r)=$ $W_{2}(r)$ ) a.e. on $[t, s]$ then $\alpha\left[Z_{1}\right](r)=\alpha\left[Z_{2}\right](r)$ (resp., $\beta\left[W_{1}\right](r)=\beta\left[W_{2}\right](r)$ ) a.e. on $[t, s]$ for every $s \in[t, T]$.

The values of the lower and upper games, called the lower and upper values are defined as follows:

Lower value of the game:

$$
V(t, x)=\inf _{\beta \in \Delta(t)} \sup _{W \in M(t)} J(t, x ; W(\cdot), \beta[W](\cdot))
$$

Upper value of the game:

$$
U(t, x)=\sup _{\alpha \in \Gamma(t)} \inf _{Z \in N(t)} J(t, x ; \alpha[Z](\cdot), Z(\cdot))
$$

The lower and upper value Isaacs equations associated with the two value functions are

$$
\left\{\begin{array}{l}
u_{t}+\langle A x, D u\rangle+F^{\mp}(t, x, D u)=0 \\
u(T, x)=h(x)
\end{array}\right.
$$

where the lower value Hamiltonian $F^{-}$is defined by

$$
F^{-}(t, x, p)=\sup _{w \in \mathscr{W}} \inf _{z \in \mathscr{Z}}\{\langle b(t, x, w, z), p\rangle+L(t, x, w, z)\}
$$

and the upper value Hamiltonian $F^{+}$is defined by 


$$
F^{+}(t, x, p)=\inf _{z \in \mathscr{Z}} \sup _{w \in \mathscr{W}}\{\langle b(t, x, w, z), p\rangle+L(t, x, w, z)\} .
$$

Above $\langle\cdot, \cdot\rangle$ is the inner product in $H$, and $D u$ is the Fréchet derivative of a function $u$.

The lower value function $V$ should be the unique viscosity solution of the lower Isaacs equation (5) ( $^{-}$(with $F^{-}$) and the upper value function $U$ should be the unique viscosity solution of the upper Isaacs equation $(5)^{+}\left(\right.$with $F^{+}$). The definition of viscosity solution will be given in the next section. Equality $F^{-}=F^{+}$is known as the Isaacs condition. If this happens, by uniqueness of viscosity solutions of (5) we obtain that $V=U$ and we then say that the game has value.

Notice that, since supinf $\leq \inf$ sup, we always have $F^{-} \leq F^{+}$. In particular if $V$ is a viscosity solution of $\left(5^{-}\right.$then it is a viscosity subsolution of $(5)^{+}$so if comparison holds for (5) (a subsolution is less than or equal to a supersolution) we get $V \leq U$.

There are several papers in which viscosity solutions and Isaacs equations have been used to construct a saddle (and an approximate saddle) point strategies for infinite dimensional differential games in Hilbert spaces [12, 17, 19]. In these papers Berkovitz's notion of strategy and pay-off [1] is employed. We show how to construct nearly optimal strategies in the Elliott-Kalton sense. Our approach is based on the proofs of sub- and superoptimality principles of dynamic programming which are interesting on their own and in fact are the main results of this paper. The proofs use the method of regularization by sup- and inf-convolutions and integration along trajectories, together with some techniques of [9] and [11]. This method was used before to show sub- and superoptimality principles for finite dimensional control problems [21] and finite dimensional stochastic differential games [22], and was recently generalized to infinite dimensional control problems [10]. Similar method was employed in [3] to construct stabilizing feedbacks for nonlinear systems and in [20] to study feedback stabilization of finite dimensional nonlinear $H_{\infty}$ control systems.

\section{Notation, definitions and background}

Throughout this paper $H$ is a real separable Hilbert space equipped with the inner product $\langle\cdot, \cdot\rangle$ and the norm $\|\cdot\|$.

Let $B$ be a bounded, linear, positive, self-adjoint operator on $H$ such that $A^{*} B$ is bounded on $H$ and

$$
\left\langle\left(A^{*} B+c_{0} B\right) x, x\right\rangle \leq 0 \quad \text { for all } x \in H
$$

for some $c_{0} \leq 0$. Such an operator always exists, for instance $B=\left((-A+I)\left(-A^{*}+\right.\right.$ $I))^{-1 / 2}$ (see [18]). We refer to [5] for various examples of $B$. Using the operator $B$ we define for $\gamma>0$ the space $H_{-\gamma}$ to be the completion of $H$ under the norm 


$$
\|x\|_{-\gamma}=\left\|B^{\frac{\gamma}{2}} x\right\| .
$$

Let $\Omega \subset[0, T] \times H$. We say that $u: \Omega \rightarrow \mathbb{R}$ is $B$-upper-semicontinuous (respectively, $B$-lower-semicontinuous) on $\Omega$ if whenever $t_{n} \rightarrow t, x_{n} \rightarrow x, B x_{n} \rightarrow B x,(t, x) \in \Omega$, then $\lim \sup _{n \rightarrow+\infty} u\left(t_{n}, x_{n}\right) \leq u(t, x)$ (respectively, $\liminf _{n \rightarrow+\infty} u\left(t_{n}, x_{n}\right) \geq u(t, x)$ ). The function $u$ is $B$-continuous on $\Omega$ if it is $B$-upper-semicontinuous and $B$-lowersemicontinuous on $\Omega$.

We will say that a function $v$ is $B$-semiconvex (respectively, $B$-semiconcave) if there exists a constant $C \geq 0$ such that $v(t, x)+C\left(\|x\|_{-1}^{2}+t^{2}\right)$ is convex (respectively, $v(t, x)-C\left(\|x\|_{-1}^{2}+t^{2}\right)$ is concave).

We will denote by $B_{R}$ the open ball of radius $R$ centered at 0 in $H$.

The following conditions will be assumed throughout the paper.

\section{Hypothesis 1}

- A is a linear, densely defined, maximal dissipative operator in $H$.

- $\mathscr{W}$ and $\mathscr{Z}$ are complete, separable metric spaces.

- $b:[0, T] \times H \times \mathscr{W} \times \mathscr{Z} \rightarrow H$ is continuous,

$$
\sup _{(t, w, z) \in[0, T] \times \mathscr{W} \times \mathscr{Z}}\|b(t, 0, w, z)\| \leq+\infty,
$$

and there exist a constant $M>0$ and a local modulus of continuity $\omega$ such that

$$
\|b(t, x, w, z)-b(s, y, w, z)\| \leq M\left\|x_{1}-x_{2}\right\|_{-1}+\omega(|t-s| ;\|x\| \wedge\|y\|)
$$

for all $t, s \in[0, T], x, y \in H, w \in \mathscr{W}, z \in \mathscr{Z}$.

- $L:[0, T] \times H \times \mathscr{W} \times \mathscr{Z} \rightarrow H$ is continuous,

$$
\begin{gathered}
|L(t, x, w, z)| \leq C\left(1+\|x\|^{k}\right) \quad \text { for some } k \geq 0, \\
|L(t, x, w, z)-L(s, y, w, z)| \leq+\omega\left(|t-s|+\|x-y\|_{-1} ;\|x\| \vee\|y\|\right)
\end{gathered}
$$

for all $t, s \in[0, T], x, y \in H, w \in \mathscr{W}, z \in \mathscr{Z}$ and some local modulus $\omega$.

The solution of the Isaacs equation is understood in a modified viscosity sense of Crandall and Lions [5, 6]. We consider two sets of tests functions:

$$
\begin{gathered}
\text { test } 1=\left\{\varphi \in C^{1}((0, T) \times H): \varphi \text { is } B\right. \text {-lower semicontinuous and } \\
\left.A^{*} D \varphi \in C((0, T) \times H)\right\}
\end{gathered}
$$

and

$$
\begin{aligned}
\text { test } 2=\left\{g \in C^{1}((0, T) \times H):\right. & \exists g_{0},:[0,+\infty) \rightarrow[0,+\infty), \\
& \text { and } \eta \in C^{1}((0, T)) \text { positive } s . t . \\
& g_{0} \in C^{1}\left([0,+\infty), g_{0}^{\prime}(r) \geq 0 \forall r \geq 0,\right. \\
& \left.g_{0}^{\prime}(0)=0 \text { and } g(t, x)=\eta(t) g_{0}(\|x\|)\right\}
\end{aligned}
$$

Sometimes it is necessary to take the finite linear combinations of functions $\eta(t) g_{0}(\|x\|)$ above as test 2 functions, however this will not be needed here. 
Below we present the definition of viscosity solution. We point out that this definition applies to terminal value problems.

Definition 1. A $B$-upper semicontinuous function $u$ on $(0, T) \times H$ is a viscosity subsolution of

$$
u_{t}+\langle A x, D u\rangle+F(t, x, D u)=0 \quad \text { in }(0, T) \times H
$$

if whenever $v-\varphi-g$ has a local maximum at $(\bar{t}, \bar{x}) \in(0, T) \times H$ for $\varphi \in$ test 1 and $g \in$ test 2 then

$$
\varphi_{t}(\bar{t}, \bar{x})+g_{t}(\bar{t}, \bar{x})+\left\langle A^{*} D \varphi(\bar{t}, \bar{x}), \bar{x}\right\rangle+F(\bar{t}, \bar{x}, D \varphi(\bar{t}, \bar{x})+D g(\bar{t}, \bar{x})) \geq 0 .
$$

A $B$-lower semicontinuous function $u$ on $(0, T) \times H$ is a viscosity supersolution of (7) if whenever $v+\varphi+g$ has a local minimum at $(\bar{t}, \bar{x}) \in(0, T) \times H$ for $\varphi \in$ test 1 and $g \in$ test 2 then

$$
-\varphi_{t}(\bar{t}, \bar{x})-g_{t}(\bar{t}, \bar{x})-\left\langle A^{*} D \varphi(\bar{t}, \bar{x}), \bar{x}\right\rangle+F(\bar{t}, \bar{x},-D \varphi(\bar{t}, \bar{x})-D g(\bar{t}, \bar{x})) \leq 0 .
$$

A function $v$ is a viscosity solution of (7) if it is both a viscosity subsolution and a viscosity supersolution of $[\mathbf{7}$.

For a function $v$ we will denote by $D^{+} v(t, x)$ the superdifferential of $v$ at $(t, x)$, i.e. the set of all pairs $(a, p) \in \mathbb{R} \times H$ such that

$$
v(s, y)-v(t, x)-\langle p, y-x\rangle-a(s-t) \leq o(\|x-y\|+|t-s|) .
$$

The subdifferential $D^{-} v(t, x)$ is defined the same by reversing the inequality above. It is well known (see for instance [2]) that for a semi-convex (resp., semi-concave) function $v$ its subdifferential (resp., superdifferential) at a point $(s, z)$ is equal to

$$
\overline{\operatorname{conv}}\left\{(a, p): v_{t}\left(s_{n}, z_{n}\right) \rightarrow a, D v\left(s_{n}, z_{n}\right) \rightarrow p, s_{n} \rightarrow s, z_{n} \rightarrow z\right\}
$$

i.e. it is equal to the closure of the convex hull of the set of weak limits of derivatives of $v$ nearby.

Solution of (1] is understood in the mild sense (see for instance [16]), which means that

$$
X(s)=e^{(s-t) A} x+\int_{t}^{s} e^{(s-\tau) A} b(\tau, X(\tau), W(\tau), Z(\tau)) d \tau
$$

It is well known [16] that under the assumptions of Hypothesis 1 for every $t \in$ $[0, T], x \in H$ there exists a unique mild solution $X \in C(t, T ; H)$ of $\mathbb{1}$. Moreover there exists a constant $C=C(T,\|x\|)$, independent of $W \in M(t)$ and $Z \in N(t)$, such that

$$
\max _{t \leq s \leq T}\|X(s)\| \leq C .
$$

Also we have that if $\varphi$ is a test 1 function, $t<\tau<T$, and $X$ is the solution of (1), then 


$$
\begin{gathered}
\varphi(\tau, X(\tau))-\varphi(t, x)=\int_{t}^{\tau}\left(\varphi_{t}(r, X(r))+\left\langle X(r), A^{*} D \varphi(r, X(r))\right\rangle\right. \\
+\langle b(r, X(r), W(r), Z(r)), D \varphi(r, X(r))\rangle) d r
\end{gathered}
$$

(see [16], Proposition 5.5, page 67).

\section{Regularization by $B$-sup- and $B$-inf-convolutions}

We first recall the results about sup- and inf-convolutions from [10]. Following the functions introduced in [6] and their modifications from [10], for a function $u$ : $(0, T) \times H \rightarrow \mathbb{R}$ and $\varepsilon, \beta, \lambda>0, m \geq 2, K \geq 0$, we define the $B$-sup-convolution of $u$ by

$$
u^{\lambda, \varepsilon, \beta}(t, x)=\sup _{(s, y)}\left\{u(s, y)-\frac{\|x-y\|_{-1}^{2}}{2 \varepsilon}-\frac{(t-s)^{2}}{2 \beta}-\lambda e^{2 m K(T-s)}\|y\|^{m}\right\}
$$

and the $B$-inf-convolution of $u$ by

$$
u_{\lambda, \varepsilon, \beta}(t, x)=\inf _{(s, y)}\left\{u(s, y)+\frac{\|x-y\|_{-1}^{2}}{2 \varepsilon}+\frac{(t-s)^{2}}{2 \beta}+\lambda e^{2 m K(T-s)}\|y\|^{m}\right\} .
$$

The following properties of $B$-sup- and inf-convolutions have been proved in [10], Lemma 4.2.

Lemma 1. Let $w$ be such that

$$
w(t, x) \leq C\left(1+\|x\|^{k}\right) \quad\left(\text { respectively, } w(t, x) \geq-C\left(1+\|x\|^{k}\right)\right)
$$

on $(0, T) \times H$ for some $k \geq 0$. Let $m>\max (k, 2)$. Then:

(i) For every $R>0$ there exists $M_{R, \varepsilon, \beta}$ such that if $v=w^{\lambda, \varepsilon, \beta}$ (respectively, $v=$ $\left.w_{\lambda, \varepsilon, \beta}\right)$ then

$$
|v(t, x)-v(s, y)| \leq M_{R, \varepsilon, \beta}\left(|t-s|+\|x-y\|_{-2}\right) \quad \text { on }(0, T) \times B_{R}
$$

(ii) The function

$$
w^{\lambda, \varepsilon, \beta}(t, x)+\frac{\|x\|_{-1}^{2}}{2 \varepsilon}+\frac{t^{2}}{2 \beta}
$$

is convex (respectively,

$$
w_{\lambda, \varepsilon, \beta}(t, x)-\frac{\|x\|_{-1}^{2}}{2 \varepsilon}-\frac{t^{2}}{2 \beta}
$$

is concave). In particular $w^{\lambda, \varepsilon, \beta}$ (respectively, $w_{\lambda, \varepsilon, \beta}$ ) is $B$-semiconvex (respectively, B-semiconcave). 
(iii) If $v=w^{\lambda, \varepsilon, \beta}$ (respectively, $v=w_{\lambda, \varepsilon, \beta}$ ) and $v$ is differentiable at $(t, x) \in(0, T) \times$

$B_{R}$ then $\left|v_{t}(t, x)\right| \leq M_{R, \varepsilon, \beta}$, and $D v(t, x)=B q$, where $\|q\| \leq M_{R, \varepsilon, \beta}$

The next lemma is just a restatement of Lemma 4.3 of [10] for Isaacs equations. Its proof is the same as the proof of Lemma 4.3 of [10]. In fact this result can be shown for general equations not necessarily related to control or game problems.

Lemma 2. Let Hypothesis $\square$ be satisfied. Let $w$ satisfy (12) and be a B-uppersemicontinuous viscosity subsolution (respectively, a B-lower-semicontinuous viscosity supersolution) of

$$
w_{t}+\langle A x, D w\rangle+F(t, x, D w)=0,
$$

where $F=F^{-}$or $F=F^{+}$. Let $m>\max (k, 2)$. If $K>0$ is big enough then for every $R, \delta>0$ there exists a non-negative function $\gamma_{R, \delta}(\lambda, \varepsilon, \beta)$, where

$$
\lim _{\lambda \rightarrow 0} \limsup _{\varepsilon \rightarrow 0} \limsup _{\beta \rightarrow 0} \gamma_{R, \delta}(\lambda, \varepsilon, \beta)=0,
$$

such that $w^{\lambda, \varepsilon, \beta}$ (respectively, $w_{\lambda, \varepsilon, \beta}$ ) is a viscosity subsolution (respectively, supersolution) of

$$
v_{t}+\langle A x, D v\rangle+F(t, x, D v)=-\gamma_{R, \delta}(\lambda, \varepsilon, \beta) \quad \text { in }(\delta, T-\delta) \times B_{R}
$$

(respectively,

$$
\left.v_{t}+\langle A x, D v\rangle+F(t, x, D v)=\gamma_{R, \delta}(\lambda, \varepsilon, \beta) \quad \text { in }(\delta, T-\delta) \times B_{R}\right)
$$

for $\beta$ sufficiently small (depending on $\delta$ ), in the sense that if $v-\psi$ has a local maximum (respectively, $v+\psi$ has a local minimum) at $(t, x)$ for a test function $\psi=\varphi+g$ then

$$
\psi_{t}(t, x)+\left\langle x, A^{*} D \psi(t, x)\right\rangle+F(t, x, D \psi(t, x)) \geq-\gamma_{R, \delta}(\lambda, \varepsilon, \beta)
$$

(respectively,

$$
\left.-\psi_{t}(t, x)-\left\langle x, A^{*} D \psi(t, x)\right\rangle+F(t, x,-D \psi(t, x)) \leq \gamma_{R, \delta}(\lambda, \varepsilon, \beta)\right) .
$$

The above lemma leaves us with a small problem. It is not even clear if $u^{\lambda, \varepsilon, \beta}$ and $u_{\lambda, \varepsilon, \beta}$ satisfy pointwise inequalities at all points of their differentiability. This is indeed true. In fact under some assumptions on the Hamiltonian $F, w^{\lambda, \varepsilon, \beta}$ satisfies the Isaacs inequality at every point for some elements of its subdifferential and $w_{\lambda, \varepsilon, \beta}$ satisfies the Isaacs inequality at every point for some elements of its superdifferential. The right elements of the sub-/superdifferentials are the weak limits of derivatives. In Lemma 3 we use the notation from Lemma 2 The proof of Lemma 3 is the same as the proof of Lemma 4.5 of [10]. 
Lemma 3. Let the assumptions of Lemma 2 be satisfied and let $F(t, x, \cdot)$ be weakly sequentially continuous for every $(t, x) \in(0, T) \times H$. Let $(a, p) \in D^{-} w^{\lambda, \varepsilon, \beta}(t, x)$ (respectively, $\left.(a, p) \in D^{+} w_{\lambda, \varepsilon, \beta}(t, x)\right)$ be such that $\left(w_{t}^{\lambda, \varepsilon, \beta}\left(t_{n}, x_{n}\right), D w^{\lambda, \varepsilon, \beta}\left(t_{n}, x_{n}\right)\right) \rightarrow$ $(a, p)$ for some $\left(t_{n}, x_{n}\right) \rightarrow(t, x)$ (respectively, $\left(\left(w_{\lambda, \varepsilon, \beta}\right)_{t}\left(t_{n}, x_{n}\right), D w_{\lambda, \varepsilon, \beta}\left(t_{n}, x_{n}\right)\right) \rightarrow$ $(a, p))$. Then

$$
a+\left\langle x, A^{*} p\right\rangle+F(t, x, p) \geq-\gamma(\lambda, \varepsilon, \beta)
$$

(respectively,

$$
\left.a+\left\langle x, A^{*} p\right\rangle+F(t, x, p) \leq \gamma(\lambda, \varepsilon, \beta)\right) .
$$

The assumption that $F^{\mp}(t, x, \cdot)$ be weakly sequentially continuous is a bit restrictive but it is satisfied for instance when $\mathscr{W}$ and $\mathscr{Z}$ are compact metric spaces.

Lemma 4. If $\mathscr{W}, \mathscr{Z}$ are compact metric spaces then $F^{-}(t, x, \cdot)$ and $F^{+}(t, x, \cdot)$ are weakly sequentially continuous for every $(t, x) \in(0, T) \times H$.

Proof. We will only show the weak sequential continuity of $F^{-}$. Let $p_{n} \rightarrow p$. Recall that $F^{-}=\sup _{w} \inf _{z}$. Therefore for every $w$ there is $z_{n}$ such that

$$
F^{-}\left(t, x, p_{n}\right) \geq\left\langle p_{n}, b\left(t, x, w, z_{n}\right)\right\rangle+L\left(t, x, w, z_{n}\right)-\frac{1}{n} .
$$

By compactness of $\mathscr{Z}$ we can assume that $z_{n} \rightarrow \bar{z}$ for some $\bar{z}$ so passing to the liminf above we get

$$
\liminf _{n \rightarrow \infty} F^{-}\left(t, x, p_{n}\right) \geq\langle p, b(t, x, w, \bar{z})\rangle+L(t, x, w, \bar{z}) .
$$

It is now enough to take the $\inf _{z}$ and then $\sup _{w}$ in the above inequality to obtain the weak sequential lower-semicontinuity of $F^{-}$. The weak sequential uppersemicontinuity of $F^{-}$is proved similarly.

\section{Sub- and superoptimality principles and construction of almost optimal strategies}

The proofs of the the following sub- and superoptimality inequalities of dynamic programming are the main result of this paper.

Theorem 1. Let Hypothesis \be satisfied and let $F^{-}(t, x, \cdot)$ and $F^{+}(t, x, \cdot)$ be weakly sequentially continuous for every $(t, x) \in(0, T) \times H$. Suppose that for every $(t, x)$ there exists a modulus $\omega_{t, x}$ such that if $X$ is the solution of (1) then

$$
\left\|X\left(s_{2}\right)-X\left(s_{1}\right)\right\| \leq \omega_{t, x}\left(s_{2}-s_{1}\right)
$$

for all $t \leq s_{1} \leq s_{2} \leq T$ and all $W \in M(t), Z \in N(t)$. Let $u$ be a function such that

$$
|u(t, x)| \leq C\left(1+\|x\|^{k}\right) \quad \text { for all } x \in H
$$


for some $k \geq 0$ and such that for every $R>0$ there exists a modulus $\sigma_{R}$ such that

$$
|u(t, x)-u(s, y)| \leq \sigma_{R}\left(|t-s|+\|x-y\|_{-1}\right)
$$

for all $t, s \in[0, T],\|x\|,\|y\| \leq R$. Then:

(i) If $u$ is a viscosity supersolution of (5) ${ }^{-}$then for every $0<t<s<T, x \in H$

$$
u(t, x) \geq \inf _{\beta \in \Delta(t)} \sup _{W \in M(t)}\left\{\int_{t}^{s} L(\tau, X(\tau), W(\tau), \beta[W](\tau)) d \tau+u(s, X(s))\right\} .
$$

(ii) If $u$ is a viscosity subsolution of (5) $)^{-}$then for every $0<t<s<T, x \in H$

$$
u(t, x) \leq \inf _{\beta \in \Delta(t)} \sup _{W \in M(t)}\left\{\int_{t}^{s} L(\tau, X(\tau), W(\tau), \beta[W](\tau)) d \tau+u(s, X(s))\right\} .
$$

(iii) If $u$ is a viscosity supersolution of (5) ${ }^{+}$then for every $0<t<s<T, x \in H$

$$
u(t, x) \geq \sup _{\alpha \in \Gamma(t)} \inf _{Z \in N(t)}\left\{\int_{t}^{s} L(\tau, X(\tau), \alpha[Z](\tau), Z(\tau)) d \tau+u(s, X(s))\right\} .
$$

(iv) If $u$ is a viscosity subsolution of $(5)^{+}$then for every $0<t<s<T, x \in H$

$$
u(t, x) \leq \sup _{\alpha \in \Gamma(t)} \inf _{Z \in N(t)}\left\{\int_{t}^{s} L(\tau, X(\tau), \alpha[Z](\tau), Z(\tau)) d \tau+u(s, X(s))\right\} .
$$

Remark 1. Condition (19) says that trajectories starting at a fixed point $x$ at a fixed time $t$ are uniformly continuous on $[t, T]$, uniformly in $W \in M(t), Z \in N(t)$. It is a little restrictive, however it seems necessary to obtain uniform estimates on the error terms in the proof. In general one may expect it to hold when the semigroup $e^{t A}$ has some regularizing properties. It was shown in [10] that it holds for example if $A=A^{*}$, it generates a differentiable semigroup, and

$$
\left\|A e^{t A}\right\| \leq \frac{C}{t^{\delta}}
$$

for some $\delta<2$. One can also check that (19) is satisfied if the semigroup $e^{t A}$ is compact.

Proof. We will only show (i) and (ii) as the proofs of (iii) and (iv) are analogous.

(i) Step 1. (Reduction to the $B$-semiconcave case): Let $(t, x)$ be fixed and let $\delta$ be such that $0<\delta<t<t+h<T-\delta$. We choose $m$ and $K$ as in Lemma2 We notice that it follows from 20 and 21 that

$$
\left|u_{\lambda, \varepsilon, \beta}(\tau, y)-u(\tau, y)\right| \leq \tilde{\sigma}_{R}(\lambda+\varepsilon+\beta) \quad \text { for } \tau \in(0, T),\|y\| \leq R,
$$

where the modulus $\tilde{\sigma}_{R}$ can be explicitly calculated from $\sigma_{R}$. Since by 10 all trajectories of (1) stay in some ball $B_{R}$ on time interval $[t, T]$, setting $L:=L-\gamma_{R, \delta}(\lambda, \varepsilon, \beta)$, 
using 14, 24 and Lemma 3 it is enough to show (ii) when $u$ is $B$-semiconcave and such that for every $(\tau, y) \in(\delta, T-\delta) \times B_{R}$ there exists $(a, p) \in D^{+} u(\tau, y)$ such that

$$
a+\left\langle y, A^{*} p\right\rangle+F^{-}(\tau, y, p) \leq 0 .
$$

By Lemma 1 we may assume that $u(\tau, y)-\frac{\|y\|_{-1}^{2}}{2 \varepsilon}-\frac{\tau^{2}}{2 \beta}$ is concave.

Step 2.(Construction of nearly optimal control): Let $W \in M(t)$ be a fixed control. For $n \geq 1$ set $h=(s-t) / n$. Take $(a, p) \in D^{+} u(t, x)$ satisfying (25). (Recall $F^{-}=$ $\sup _{w} \inf _{z}$.) It then follows from the separability of $\mathscr{W}$ and the continuity of $b$ and $L$ that there exist points $z_{1}, z_{2}, \ldots \in \mathscr{Z}, w_{1}, w_{2}, \ldots \in \mathscr{W}$ and balls $B_{r_{i}}\left(w_{i}\right), i=1,2, \ldots$ such that $\cup_{i=1}^{\infty} B_{r_{i}}\left(w_{i}\right)=\mathscr{W}$ and

$$
a+\left\langle x, A^{*} p\right\rangle+\left\langle b\left(t, x, w, z_{i}\right), p\right\rangle+L\left(t, x, w, z_{i}\right) \leq \frac{1}{n}
$$

for $w \in B_{r_{i}}\left(w_{i}\right)$. Define a map $\Psi: \mathscr{W} \rightarrow \mathscr{Z}$ by

$$
\Psi(w)=z_{i} \quad \text { if } w \in B_{r_{i}}\left(w_{i}\right) \backslash \bigcup_{j=1}^{i-1} B_{r_{j}}\left(w_{j}\right), i=1,2, \ldots
$$

Define a control $Z^{n} \in N(t)$ by

$$
Z^{n}(\tau)=\Psi(W(\tau)) \text { for } \tau \in[t, t+h) .
$$

Then

$$
a+\left\langle x, A^{*} p\right\rangle+\left\langle b\left(t, x, W(\tau), Z^{n}(\tau)\right), p\right\rangle+L\left(t, x, W(\tau), Z^{n}(\tau)\right) \leq \frac{1}{n}
$$

for $\tau \in[t, t+h)$. Denote by $X(\tau)$ the trajectory corresponding to controls $W(\tau)$ and $Z^{n}(\tau)$.

Step 3. (Integration along trajectories): It follows from the $B$-semiconcavity of $u$ that

$$
u(\tau, y) \leq u(t, x)+a(\tau-t)+\langle p, y-x\rangle+\frac{\|y-x\|_{-1}^{2}}{2 \varepsilon}+\frac{(\tau-t)^{2}}{2 \beta} .
$$

Therefore it follows from 11, 19, and (26) that

$$
\begin{aligned}
u(t+ & h, X(t+h)) \leq u(t, x)+\int_{t}^{t+h}\left[a+\left\langle X(\tau), A^{*}\left(p+\frac{B(X(\tau)-x)}{\varepsilon}\right)\right\rangle\right. \\
& \left.+\left\langle b\left(\tau, X(\tau), W(\tau), Z^{n}(\tau)\right), p+\frac{B(X(\tau)-x)}{\varepsilon}\right\rangle\right] d \tau+\frac{h^{2}}{2 \beta} \\
\leq & u(t, x)+\int_{t}^{t+h}\left[a+\left\langle X(\tau), A^{*} p\right\rangle+\left\langle b\left(\tau, X(\tau), W(\tau), Z^{n}(\tau)\right), p\right\rangle\right] d \tau+o\left(\frac{1}{n}\right) \\
\leq & u(t, x)+\int_{t}^{t+h}\left[a+\left\langle x, A^{*} p\right\rangle+\left\langle b\left(t, x, W(\tau), Z^{n}(\tau)\right), p\right\rangle\right] d \tau+o\left(\frac{1}{n}\right)
\end{aligned}
$$




$$
\begin{aligned}
\leq & u(t, x)-\int_{t}^{t+h} L\left(t, x, W(\tau), Z^{n}(\tau)\right) d \tau+o\left(\frac{1}{n}\right) \\
& \leq u(t, x)-\int_{t}^{t+h} L\left(\tau, X(\tau), W(\tau), Z^{n}(\tau)\right) d \tau+o\left(\frac{1}{n}\right),
\end{aligned}
$$

where $o\left(\frac{1}{n}\right)$ is independent of $W$ and $\Psi$. We now repeat the above process starting at $\left(t+h, X(t+h)\right.$ to obtain a control $Z_{n}$ on $[t+h, t+2 h)$ such that

$u(t+2 h, X(t+2 h)) \leq u(t+h, X(t+h))-\int_{t+h}^{t+2 h} L\left(\tau, X(\tau), W(\tau), Z^{n}(\tau)\right) d \tau+o\left(\frac{1}{n}\right)$.

After $n$ iterations we produce a control $Z_{n}$ on $[t, s)$ which we can extend to the interval $[t, T]$ so that $Z_{n} \in N(t)$ for which

$$
u(s, X(s)) \leq u(t, x)-\int_{t}^{s} L\left(\tau, X(\tau), W(\tau), Z^{n}(\tau)\right) d \tau+n o\left(\frac{1}{n}\right)
$$

where $o\left(\frac{1}{n}\right)$ is independent of $W$ and $Z^{n}$

Step 4. (Construction of the strategy): We now define a strategy

$$
\beta^{n}[W](\tau):=Z^{n}(\tau)
$$

By construction of $Z_{n}$ it is clear that $\beta^{n}$ is nonanticipating, i.e. $\beta^{n} \in \Delta(t)$. Moreover we have

$$
u(t, x) \geq \int_{t}^{s} L\left(\tau, X(\tau), W(\tau), \beta^{n}[W](\tau)\right) d \tau+u(s, X(s))+n o\left(\frac{1}{n}\right)
$$

where $o\left(\frac{1}{n}\right)$ is independent of $W$ and $\beta^{n}$. This gives the superoptimality principle (22) after we take the sup over $W \in M(t)$, then inf over $\beta \in \Delta(t)$, and then let $n \rightarrow \infty$.

(ii) Let $(t, x)$ be fixed and let $\delta$ be such that $0<\delta<t<t+h<T-\delta$. By the same argument as in Step 1 of $(i)$ it is enough to show $(i i)$ when $u$ is $B$-semiconcave and is such that for every $(\tau, y) \in(\delta, T-\delta) \times B_{R}$ (for $R$ big enough) there exists $(a, p) \in D^{-} u(\tau, y)$ such that

$$
a+\left\langle y, A^{*} p\right\rangle+F^{-}(\tau, y, p) \geq 0 .
$$

Let $Z$ be any control in $N(t)$. For $n \geq 1$ we set $h=(s-t) / n$. We take $(a, p) \in$ $D^{-} u(t, x)$ satisfying 27. and we choose $w_{1} \in \mathscr{W}$ such that

$$
a+\left\langle x, A^{*} p\right\rangle+\inf _{z \in \mathscr{Z}}\left\{\left\langle b\left(t, x, w_{1}, z\right), p\right\rangle+L\left(t, x, w_{1}, z\right)\right\} \geq-\frac{1}{n},
$$

and then we define a control $W^{n}(\tau)=w_{1}$ for $\tau \in[t, t+h)$ Arguing similarly as in Step 2 of $(i)$ we then obtain 


$$
u(t+h, X(t+h)) \geq u(t, x)-\int_{t}^{t+h} L\left(\tau, X(\tau), W^{n}(\tau), Z(\tau)\right) d \tau+o\left(\frac{1}{n}\right)
$$

where $X$ is the solution of 11 with the controls $W^{n}$ and $Z$, and the term $o\left(\frac{1}{n}\right)$ is independent of $Z$ and $w_{1}$. After $n$ iterations of this process we produce points $w_{1}, \ldots, w_{n} \in \mathscr{W}$ and a piecewise constant control $W^{n} \in M(t)$ such that $w^{n}(\tau)=w_{i}$ for $\tau \in[t+(t-1) h, t+i h), i=1, \ldots, n$, and such that

$$
u(s, X(s)) \geq u(t, x)-\int_{t}^{s} L\left(\tau, X(\tau), W^{n}(\tau), Z(\tau)\right) d \tau+n o\left(\frac{1}{n}\right),
$$

where $o\left(\frac{1}{n}\right)$ is independent of $Z$ and $W^{n}$ by 19 . Therefore we can define a nonanticipative strategy $\alpha^{n} \in \Gamma(t)$ by setting $\alpha^{n}[Z](\tau)=W^{n}(\tau)$. This strategy satisfies

$$
u(s, X(s)) \geq u(t, x)-\int_{t}^{s} L\left(\tau, X(\tau), \alpha^{n}[Z](\tau), Z(\tau)\right) d \tau+n o\left(\frac{1}{n}\right)
$$

where $o\left(\frac{1}{n}\right)$ is independent of $Z$ and the definition of $\alpha^{n}$. Moreover $\alpha^{n}[Z]_{\mid[t+(i-1) h, t+i h)}$ depends only on $Z_{\mid[t, t+(i-1) h)}$ for $i=1, \ldots, n$.

It is now standard to notice (see [22]) that for every $\beta \in \Delta(t)$ there exist $\tilde{W} \in M(t)$ and $\tilde{Z} \in N(t)$ such that $\alpha^{n}[\tilde{Z}]=\tilde{W}$ and $\beta[\tilde{W}]=\tilde{Z}$ on $[t, s]$. We recall how to do this for reader's convenience. We first set $\tilde{W}_{\mid[t, t+h)}=w_{1}$ as $w_{i}$ only depended on $(t, x)$, and then define $\tilde{Z}_{\mid[t, t+h)}=\beta[\tilde{W}]_{\mid[t, t+h)}$. (This means that $\tilde{W}$ has to be extended to $[t, T]$ but since $\beta[\tilde{W}]_{\mid[t, t+h)}$ only depends on $\tilde{W}_{\mid[t, t+h)}$ we will not worry about this.) We proceed inductively. Suppose we know $\tilde{W}$ and $\tilde{Z}$ on $[t, t+i h)$. By the construction of $\alpha^{n}$ we now set $\tilde{W}_{\mid[t, t+(i+1) h)}=\alpha^{n}[\tilde{Z}]_{\mid[t, t+(i+1) h)}$ (since $\tilde{W}_{\mid[t, t+(i+1) h)}$ only depends on $\tilde{Z}_{\mid[t, t+i h)}$ ) and then define $\tilde{Z}_{\mid[t, t+(i+1) h)}=\beta[\tilde{W}]_{\mid[t, t+(i+1) h)}$. It is clear from this construction that after $n$ iterations we obtain $\alpha^{n}[\tilde{Z}]=\tilde{W}$ and $\beta[\tilde{W}]=\tilde{Z}$ on $[t, s]$.

Therefore, (28) applied to $\tilde{Z}$ gives us that for every $\beta \in \Delta(t)$ there exists $\tilde{W} \in$ $M(t)$ such that

$$
u(t, x) \leq \int_{t}^{s} L(\tau, X(\tau), \tilde{W}(\tau), \beta[\tilde{W}](\tau)) d \tau+u(s, X(s))+n o\left(\frac{1}{n}\right)
$$

where $o\left(\frac{1}{n}\right)$ is independent of $\beta$ and $\tilde{W}$. (We remind that $X$ above is the solution of 11 with $W:=\tilde{W}$ and $Z:=\beta[\tilde{W}]$.) It is now enough to take the sup over $W \in M(t)$, then inf over $\beta \in \Delta(t)$, and then let $n \rightarrow \infty$.

An immediate corollary of Theorem 1 is that viscosity solutions of $(5)^{-}$and $(5)^{+}$ must be the lower and upper value functions respectively.

Corollary 1. Let the assumptions of Theorem $\square$ be satisfied and let $u(T, x)=g(x)$ for $x \in H$. If $u$ is a viscosity solution of (5) (resp., (5) $\left.{ }^{+}\right)$then $u=V($ resp., $u=U)$. In particular $V$ and $U$ satisfy the Dynamic Programming Principle. 
Unfortunately the general proof of existence of viscosity solutions of (5) ${ }^{-}$and $(5)^{+}$is based on the Dynamic Programming Principle and the direct verification that the value function is a viscosity solution. Proofs by Perron's method with different definitions of solutions are in [13] 24] and in [14] for $B$-continuous viscosity solutions when $A$ is more coercive. When $B$ is compact existence of solutions of general equations like (1) was shown in [5] by finite dimensional approximations. Proofs using the value functions of the games are in [15 23] and in [4] when $A=0$. Unfortunately none of these papers provides an exact reference to the fact that lower and upper value functions considered in this paper are viscosity solutions of (5) $)^{-}$and (5) ${ }^{+}$in the sense of Definition 1 However the proof of this basically follows the arguments of the finite dimensional proof of [9] with necessary modifications using continuous dependence estimates for (11) and other techniques that can be found in [5] 6] 16]. The reader can also consult [15] for a complete proof in the infinite horizon case even though it uses a different definition of viscosity solution. Here we just state the result.

Theorem 2. Let Hypothesis $\square$ hold and let $h$ be a function such that

$$
|h(x)| \leq C\left(1+\|x\|^{k}\right) \quad \text { for all } x \in H
$$

for some $k \geq 0$, and such that for every $R>0$ there exists a modulus $\omega_{R}$ such that

$$
|h(x)-h(y)| \leq \omega_{R}\left(\|x-y\|_{-1}\right) \quad \text { if }\|x\|,\|y\| \leq R .
$$

Then $U$ and $V$ are the unique viscosity solutions of (1D) satisfying (20) and (21).

The proofs of the superoptimality principles $(i)$ and (iii) provide an explicit method of construction of almost optimal strategies using the Isaacs equations. Let us for instance explain how to construct for $v>0$ a $v$-optimal strategy on an interval $[t, s]$ for player II in the lower game. We apply the proof of superoptimality principle $(i)$ to the lower value function $V$. We first choose $R>\sup _{t \leq \tau \leq s}\|X(\tau)\|$ and take $\lambda, \varepsilon, \beta$ small such that

$$
\left|V(\tau, y)-V_{\lambda, \varepsilon, \beta}(\tau, y)\right| \leq \frac{v}{4} \quad \text { for } \tau \in(0, T),\|y\| \leq R
$$

and $(s-t) \gamma_{R, \delta}(\lambda, \varepsilon, \beta)<v / 4$. We then take $n$ big enough and proceed as in Steps 2-4 of the proof of $(i)$ to obtain a strategy $\beta^{n} \in \Delta(t)$ such that for every $W \in M(t)$

$$
\begin{aligned}
& \frac{v}{4}+V_{\lambda, \varepsilon, \beta}(t, x) \\
& \quad \geq \int_{t}^{s}\left(L\left(\tau, X(\tau), W(\tau), \beta^{n}[W](\tau)\right)-\gamma_{R, \delta}(\lambda, \varepsilon, \beta)\right) d \tau+V_{\lambda, \varepsilon, \beta}(s, X(s)) .
\end{aligned}
$$

This implies that

$$
v+V(t, x) \geq \int_{t}^{s} L\left(\tau, X(\tau), W(\tau), \beta^{n}[W](\tau)\right) d \tau+V(s, X(s)),
$$


i.e. $\beta^{n}$ is $v$-optimal on the interval $[t, s]$. It is a feedback strategy in a sense that $\beta^{n}[W](\tau)$ for $t+i h \leq \tau<t+(i+1) h, i=0,1, \ldots, n-1$, depends on $W$ and on $X(t+$ $i h)$, where $h=(s-t) / n$.

Remark 2. Under a different set of assumptions which guarantee that value functions for the games with $A$ replaced by bounded approximating operators (for instance the Yosida approximations or operators considered in [5]) converge to the original value functions, the same procedure can be used to construct nearly optimal feedback strategies for the approximating games with bounded operators and consequently for the original game. When the operator $A$ is bounded, the assumption (19) about equicontinuity of the trajectories is automatically satisfied.

Acknowledgements A. Święch was supported by NSF grant DMS 0500270.

\section{References}

1. L. Berkovitz, The existence of value and saddle point in games of fixed duration, SIAM J. Control Optim. 23 (1985), no. 2, 172-196. Erratum and addendum: SIAM J. Control Optim. 26 (1988), no. 3, 740-742.

2. J. M. Borwein and D. Preiss, A smooth variational principle with applications to subdifferentiability and to differentiability of convex functions, Trans. Amer. Math. Soc. 303 (1987), no. 2, 517-527.

3. F. H. Clarke, Yu. S. Ledyaev, E. D. Sontag and A. I. Subbotin, Asymptotic controllability implies feedback stabilization, IEEE Trans. Automat. Control 42 (1997), no. 10, 1394-1407.

4. M. G. Crandall and P. L. Lions, Hamilton-Jacobi equations in infinite dimensions. II. Existence of viscosity solutions, J. Funct. Anal. 65 (1986), no. 3, 368-405.

5. M. G. Crandall and P. L. Lions, Hamilton-Jacobi equations in infinite dimensions. IV. Hamiltonians with unbounded linear terms, J. Funct. Anal. 90, (1990), 237-283.

6. M. G. Crandall and P. L. Lions, Hamilton-Jacobi equations in infinite dimensions. V. Unbounded linear terms and B-continuous solutions, J. Funct. Anal. 97, (1991), 417-465.

7. M. G. Crandall and P. L. Lions, Hamilton-Jacobi equations in infinite dimensions. VI. Nonlinear $A$ and Tataru's method refined, Evolution equations, control theory, and biomathematics (Han sur Lesse, 1991), pp. 51-89, Lecture Notes in Pure and Appl. Math., 155, Dekker, New York, 1994.

8. R. J. Elliott and N. J. Kalton, The existence of value in differential games, Memoirs of the American Mathematical Society, No. 126. American Mathematical Society, Providence, R.I., 1972.

9. L. C. Evans and P. E. Souganidis, Differential games and representation formulas for solutions of Hamilton-Jacobi equations, Indiana Univ. Math. J. 33 (1984), 773-797.

10. G. Fabbri, F. Gozzi and A. Święch, Verification theorem and construction of $\varepsilon$-optimal controls for control of abstract evolution equations, to appear in J. Convex Anal..

11. W. H. Fleming and P. E. Souganidis, On the existence of value functions of two-player, zerosum stochastic differential games, Indiana Univ. Math. J. 38, No. 2 (1989), 293-314.

12. M. K. Ghosh, and A. J. Shaiju, Existence of value and saddle point in infinite-dimensional differential games, J. Optim. Theory Appl. 121 (2004), no. 2, 301-325.

13. H. Ishii, Viscosity solutions for a class of Hamilton-Jacobi equations in Hilbert spaces, J. Funct. Anal., 105 (1992), pp. 301-341.

14. D. Kelome and A. Święch, Perron's method and the method of relaxed limits for "unbounded" PDE in Hilbert spaces, Studia Math. 176 (2006), no. 3, 249-277. 
15. M. Kocan, P. Soravia and A. Święch, On differential games for infinite-dimensional systems with nonlinear, unbounded operators, J. Math. Anal. Appl. 211 (1997), no. 2, 395-423.

16. X. Y. Li and J.M. Yong, Optimal control theory for infinite-dimensional systems, Birkhauser Boston, Cambridge, MA, 1995.

17. M. Ramaswamy and A. J. Shaiju, Construction of approximate saddle point strategies for differential games in a Hilbert space, J. Optim. Theory Appl. 141 (2009), no. 2, 349-370.

18. M. Renardy, Polar decomposition of positive operators and problem od Crandall and Lions, Appl. Anal. 57-3 (1995), 383-385

19. A. J. Shaiju, Infinite horizon differential games for abstract evolution equations, Comput. Appl. Math. 22 (2003), no. 3, 335-357.

20. P. Soravia, Feedback stabilization and $H^{\infty}$ control of nonlinear systems affected by disturbances: the differential games approach, Dynamics, bifurcations, and control (Kloster Irsee, 2001), 173-190, Lecture Notes in Control and Inform. Sci., 273, Springer, Berlin, 2002.

21. A. Święch, Sub-and superoptimality principles of dynamic programming revisited, Nonlinear Anal. 26 (1996), no. 8, 1429-1436.

22. A. Święch, Another approach to the existence of value functions of stochastic differential games, J. Math. Anal. Appl. 204 (1996), no. 3, 884-897.

23. D. Tataru, Viscosity solutions of Hamilton-Jacobi equations with unbounded linear terms, J. Math. Anal. Appl. 163 (1992), pp. 345-392.

24. D. Tataru, Viscosity solutions for Hamilton-Jacobi equations with unbounded nonlinear term: a simplified approach, J. Differential Equations 111 (1994), no. 1, 123-146. 